\title{
A subjective review of perceptions about health benefits and risks associated with oily fish intake in women of child bearing age
}

\author{
J. M. McKenzie, S. Drummond and K. Ryan \\ Queen Margaret University, Musselburgh, EH21 6UU
}

Current UK recommendations for oily fish are based on considerations of both the benefits and risks of their inclusion in the diet, in relation to long-term health ${ }^{(1)}$. Consumption of long-chain omega-3 fatty acids (DHA \& EPA), found primarily in oily fish, have been shown to be beneficial in pregnancy outcome and foetal development and therefore are of particular importance for women of child bearing age ${ }^{(2)}$. Despite this, intakes of oily fish in this population remain well below the recommendation of $1-2$ portions per week (approx. $140 \mathrm{~g} / \mathrm{wk}$ ) at only $56 \mathrm{~g} / \mathrm{wk}^{(3)}$. There are many factors which have been suggested to influence intakes of oily fish ${ }^{(4)}$ ), ranging from perceptions about cost, lack of familiarity in how to prepare fish and dislike of the strong taste and odours, however little is known about attitudes and barriers in pregnant women or those of child-bearing age. This information is pivotal in developing effective campaigns to improve intakes. Therefore, the aim of this project was to review perceptions about health benefits and risk associated with the consumption of oily fish in women of child-bearing age.

A self-administered questionnaire was developed and distributed to women of child-bearing age. As well as collecting demographic information including child-bearing history, the questionnaire investigated the motivations, barriers, assessed awareness of advisories, including health benefits and specific risks which may influence fish consumption in this population. Both closed and open-ended questions were used to identify the frequency of key influencing factors and awareness was scored according to predetermined criteria to determine no or poor awareness against moderate or good awareness. A chi-squared test was used to assess potential differences in awareness of the risks in relation to motherhood.

Thirty-seven women of child-bearing age completed the questionnaire, with a mean(SD) age of 30.2(5.9) years. Six participants (16\%) exhibited moderate or good awareness of recommendations for fish consumption. Although 34 participants (92\%) were able to describe the health benefits of consuming fish, only 17 (46\%) were aware of the risks applicable to women of child-bearing age. The two dominant factors positively influencing fish consumption related to health benefits and liking the taste. Factors inhibiting the consumption of fish included the expense, the adverse smell, the chance of bones and dislike of preparing it. Mothers had significantly better awareness of potential risks than women without children $(p=0.03)$.

In conclusion, although there appears to be a good level of awareness about health benefits and this is considered a motivating factor for fish consumption, there are a wide range of factors inhibiting the consumption of fish. In addition, knowledge of the actual guidelines is lacking, as is awareness of the risks specifically associated with women of child-bearing age. Addressing this lack of awareness is fundamental in ensuring women of child bearing age maintain appropriate intakes of long-chain omega-3 fatty acids at this critical time.

1. Scientific Advisory Committee on Nutrition/Committee On Toxicology (2004) Advice on fish consumption: benefits and risks. London: The Stationary Office.

2. Jordon RG (2011). J Midwifery Womens Health 55, 520-528.

3. Bates B, Lennox A, Prentice A et al. (2011) National Diet and Nutrition Survey: Year 3 Report. London: The Stationary Office.

4. Adolphus K \& Baic S (2011). J Hum Nutr Diet 24: 375. 